\title{
Stabilising the construct of health related quality of life: 1970-2007
}

\section{David Armstrong}

This paper describes the process by which the construct of health related quality of life was stabilised as an important goal of medicine. From its initial rhetorical use in the 1970s through periods of advocacy and methodological development the construct was finally stabilised through the appearance and application of formal quality of life instruments in medical research and clinical practice. Yet despite the centrality of quality of life measurement for modern medicine and its current operationalisation in over a thousand questionnaires, the underlying construct remains contested and can still be described as a 'loose concept'.

Keywords: quality of life, medicine, loose concepts

During the second half of the $20^{\text {th }}$ century there has been a flood of new medical technologies that have transformed many aspects of clinical practice. From novel pharmaceuticals through to developments in bioengineering and the new genetics, innovative health technologies (IHTs) have had a major impact on medical diagnosis and therapy (Brown and Webster, 2004). However, the process of introducing and establishing IHTs involves more than simply the discovery or invention of a 'technical' or instrumental solution to a clinical problem: before a new medical technology can be applied in everyday health care it has to undergo some sort of testing to ensure that it achieves what it claims to achieve. Indeed, this process of technology evaluation has assumed greater and greater significance in the light of the rapidity of technological innovation, the frequent failure of past technologies to deliver their promise, and the need to apply limited health care resources to best effect.

Allocation of health care resources, whether at the level of the health care system, the clinic or the individual patient, has depended on an implicit or explicit outcome measure that would show the value of the intervention in question. Earlier in the $20^{\text {th }}$ century outcomes were largely measured in terms of mortality or 'cure'. But then two factors began to undermine the value of these traditional 
medical criteria. One was the diminishing returns in certain areas of therapy. For example, new interventions to treat many cancers and leukaemia began to show smaller and smaller effects on longevity. The other was the observation that the biological status of the patient might not reflect their subjective status, meaning that a patient might be helped biologically but report no improvement, or conversely, might achieve no biological improvement yet report considerable subjective betterment. At its most salient this was marked by interventions that produced improvements in life expectancy counter-balanced by major decline in patients' subjective sense of well-being. Indeed, post-war technological 'successes' such as renal dialysis and organ transplantation were often achieved at considerable personal cost to the patient (Le Fanu,1999)

In the 1970s a rhetoric emerged that stressed a new goal for medicine, that of improving health-related quality of life (QoL) (Armstrong and Caldwell, 2002) and over the following decade a number of existing measure - of disability, of mental health, of social functioning and of symptoms-were conscripted to the task of generating QoL measures (Armstrong et al., 2007). Of course this process was not achieved overnight. The very construct of QoL had to be sold to a sceptical profession and creative ways of capturing the concept with an empirical measure had to be sought. This process was a contested one in terms of resistance to the idea and competition between alternative and competing measures, as described below, but following its identification and promotion in the 1970s QoL has come to dominate thinking about health care outcomes. QoL measurement has grown from virtually nothing in the early 1970s to a vast literature and nearly 1,000 instruments 35 years later. Some indication of the size of the field can be established from the number of papers in the medical database PubMed that address QoL issues (Figure 1) which now total almost 100,000 references.

The process of establishing and organising this field of endeavour is continuing but the stages of development so far provide an insight into the way QoL

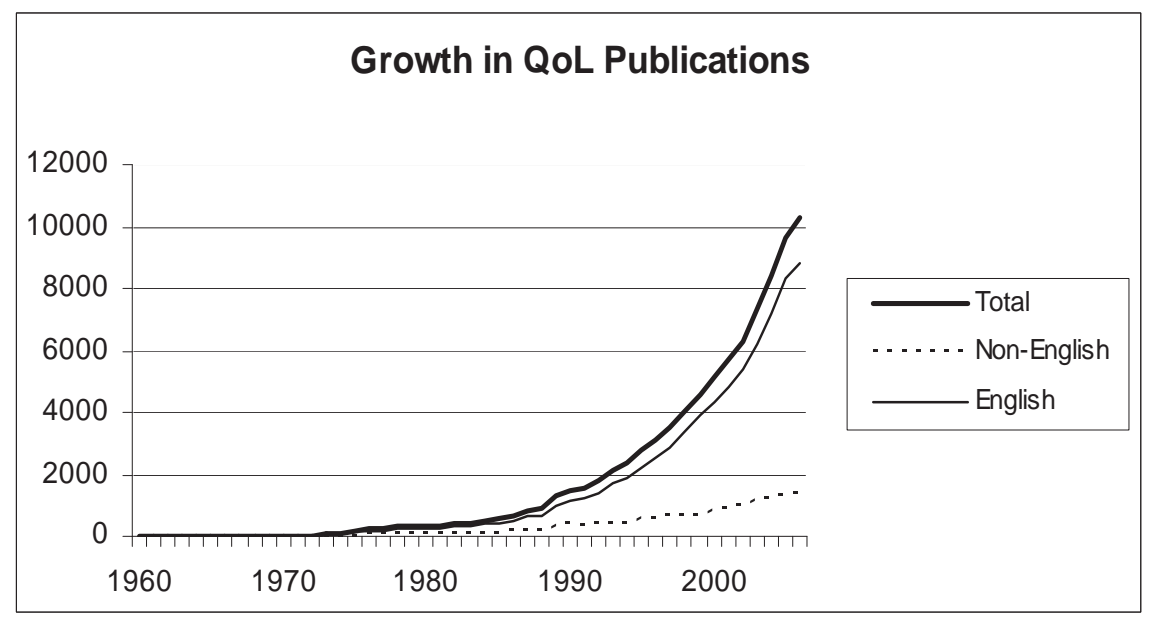

Figure 1. Growth in QoL Publications. 
'colonised' modern medicine. By turning a vague idea into a measurable 'fact' QoL made the transition from rhetorical concept to hard end-point of clinical practice. Further, while the stages of any scale development are now formalised and well described in methodological texts, the wider context of the social activity surrounding the creation of new instruments can be illustrated by the example of QoL. And while the timescale for the development of a new instrument might be measured in months or years the steps from conceptualisation to widespread implementation can take decades, as this case history illustrates.

\section{Method}

Papers dealing with QoL were identified from the PubMed database and a random sample of 50 papers was chosen from every $5^{\text {th }}$ year starting in 1980 . The number of papers available in the very first period of interest, 1975, was only 36 so a full census of these papers was taken. In total 336 papers were examined for this study.
Details of each paper were catalogued. This was mainly from the abstract but where this was missing or unclear the actual paper was examined. Papers were initially broadly classified into four categories. These were: Outcome or Empirical papers that reported QoL assessments of particular groups of patients; Review papers that collated and analysed the empirical data from papers reporting outcome data; Methodological papers that were concerned with further development of measurement procedures; and finally, Non-empirical or discursive papers that did not fit any of the above three categories. The latter, as will be shown, were much more important in the early years. The distribution of papers according to these four groups is shown in Figure 2.

Classification of papers was not always clear-cut and the changes over time suggested in Figure 2 are indicative only. They do, however, provide a useful framework for mapping the emergence of the QoL literature by showing the growth and stability of different types of paper. This broad picture can be further

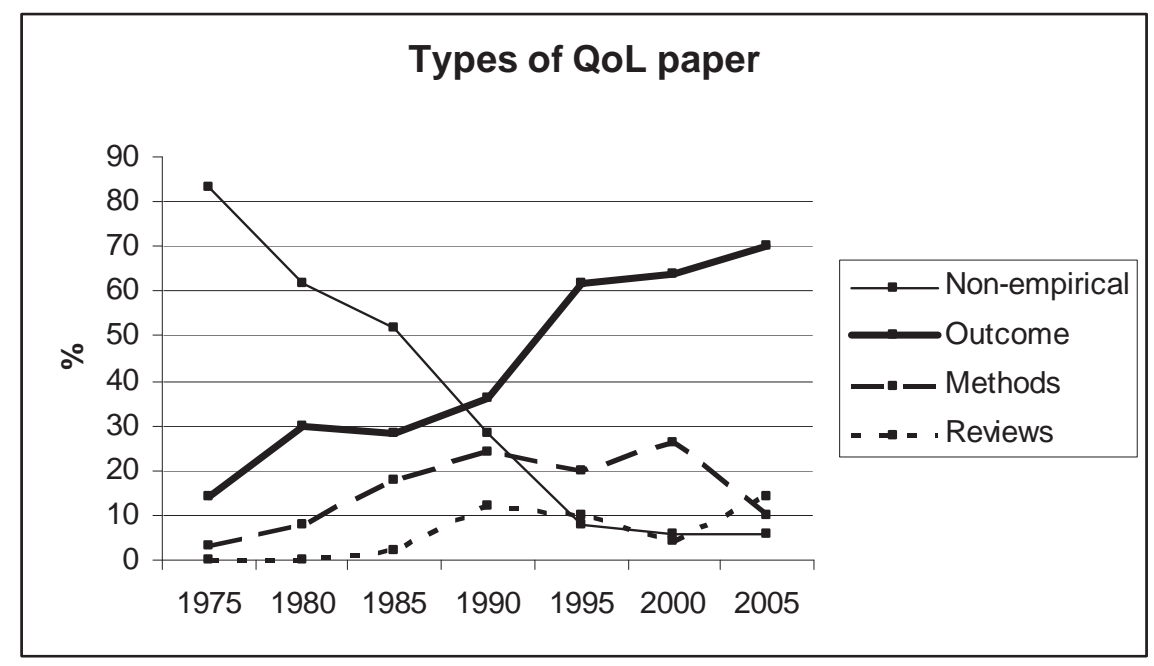

Figure 2. Proportion of different types of QoL publications 
enriched by considering changes over time within the four types of paper.

Finally database searches were also made for some of the more significant QoL measures to explore the life trajectory of individual questionnaires or instruments.

\section{Beginnings}

The first year investigated in the 5-year samples from the PubMed database, 1975 , was at the very beginning of the emergence of QoL as a major field of study and, as noted, only consisted of 36 papers in total. The dominant type of paper was the Non-empirical variety. The period might be seen as belonging to an 'advocacy' phase of development in that most papers promoted the value of taking QoL seriously as a medical outcome even though there were few empirical instances of its actual use as a health status measure. Indeed, three of the 36 papers had an explicit educational focus as they sought to persuade the reader of the importance of QoL.

Explicit advocacy has not been a strong genre in medical journals in the late $20^{\text {th }}$ century and mainly occurs in editorials. Yet of the 30 'advocacy' papers identified here only one was an editorial. A few papers were simply in the format of published lectures but most were basic appeals or exhortations to use, aim for or value the patient's QoL as a central component of health care. In consequence, given the contemporary empirical slant of most mainstream medical journals this meant that a high proportion of those papers were published in non-medical, particularly nursing (seven articles), or lower profile medical journals.

Of the remaining six papers, five were concerned with QoL as an Outcome in which empirical findings were reported. These included QoL in patients with leukaemia, with childhood cancer, on haemodialysis and as a result of improved housing. This list resonates with the medical and social debates that underpinned the emergence of the concept of QoL in that it echoes some of the 'social indicator' research as well as focuses on diseases given 'aggressive' medical therapy (Armstrong and Caldwell, 2002; Le Fanu, 1999). Two strategies were pursued in measuring QoL. One was to recruit existing measures of related concepts to the task. For example, one paper examined the effect of housing on morale and life satisfaction using two existing questionnaires and another argued that as 'sense of security' was an aspect of QoL it was then justified to use 'fear' as a negative correlate of QoL in a secondary data analysis. The other strategy was to create new rudimentary measures such as assessing whether QoL was good, fair or poor supplemented by assessments of loss of income and family life.

There was only one paper that explicitly addressed methodological issues-in this case a review of procedures for valuing lives. Even so, two of the Non-empirical papers discussed the nature of QoL which might be considered a conceptual precursor to the measurement process.

In summary the 1970s marked the birth of health-related QoL as a legitimate medical outcome. Mainly, published papers were concerned with 'marketing' the idea of QoL as a goal worth pursuing even though at the time ways of measuring the concept were few and rudimentary. Once the idea was accepted, however, this deficit could be rectified with a surge in methodology-driven papers creating and validating new instruments and reports of their empirical application. 


\section{The decline of Non-empirical papers}

Whereas 90\% of papers in 1975 were Nonempirical rhetorical or opinion pieces, five years later the proportion had fallen to nearer $50 \%$ (28 out of 50 ). Moreover, the content of these papers had begun to change: instead of arguments for the importance of QoL the agenda was how to measure it and apply it. Given this focus several of these papers could be described as quasi-methodological; they did not address measurement issues directly but they began the conceptual ground-clearance that would enable the emergence of formal instruments. The involvement of nursing journals that had been such a feature of the 1975 literature, however, began to decline - a process that was speed up during the decade such that by the 1990s nursing journals contributed only marginally to the QoL debates.

The main focus of the Nonempirical papers remained advocacy and persuasion with a wider range of medical conditions being described that would benefit from applying QoL as the principal outcome of care. But there was also a new thread in seven out of the 28 Non-empirical papers as the recently emergent medical ethics or bioethics began to engage with the QoLdebate (with ethical, legal and administrative journals provided a good proportion of this type of paper) (Callahan, 1973; Jonsen, 1998; Martensen, 2001; Rothman, 1991). In life and death decisions in particular QoL began to figure as the reference state for ethical judgement.

In 1985 the proportion of Nonempirical papers held steady at 24 out of 50. An increasing proportion of these were in ethics and/or legal journals with a declining number in nursing journals. Most of these papers remained polemical but two new genres can be identified as beginning to emerge. The first was greater theoretical dissection of QoL (that also overlapped with the Methodological papers). As new questionnaires were devised and QoL ratings for patients' experience accumulated (see below) it became necessary to question what exactly was being measured: empirical developments thereforeled to evengreater scrutiny of conceptual underpinnings. The second new genre of papers was an increasing emphasis on educating the reader about the substantive parts of the QoL movement. The latter especially signified a more confident shift from discussing what should happen to what was happening.

In the 1990s the proportion of Nonempirical papers began a rapid decline. In 1990, for example, their proportion halved to only $28 \%$ of that year's sample. Over a third of these were now explicitly educational in nature while papers concerned with ethical aspects of QoL had declined to only two papers. And by 1995 Non-empirical papers had shrunk to only four (8\%). One of these concerned ethics, the other three were editorials offering an assessment of QoL in a particular area, though not formally a review paper. In both 2000 and 2005 Nonempirical papers were down to three papers mainly 'educational/review' type editorials.

Thus some 20 years after the appearance of QoL as a concept and measure in medicine the discursive 'opinion' piece that had extolled its virtues or promoted its uptake had virtually come to an end. Given the contemporary growth in empirical and methodological papers this might be held to mark the end of the struggle to get QoL accepted. There was now no need to exhort colleagues to take an interest in this 'new' idea as it was 
firmly established. What it needed next was more work on its measurement and studies to show its value.

\section{The rise of Outcomes research}

Whereas in 1975 only five out of 36 papers presented empirical evidence of QoL in specific clinical populations, by 1980 fi fteen papers out of the 50 sampled presented QoL as an empirical outcome measure. The commonest approach (8 out of 15) was simply to describe the QoL in a cross-section of patients with a specific condition. The implicit justification seemed to lie in the choice of medical conditions which all in some way had high impact on QoL. The QoL of patients with cystic fibrosis, haemodialysis and ileostomy was all believed to be impaired, the question was by how much.

Three out of the 15 empirical papers reported QoL before and after a medical intervention (radiation for prostate cancer and mastectomy for breast cancer), two compared the impacts of different treatments on QoL (renal transplant versus dialysis and the QoL of volunteers and non-volunteers) and one used QoL as an outcome in a randomised clinical trial (of cytotoxic drugs versus endocrine therapy in breast cancer). The way QoL was measured in these empirical studies varied widely from use of the formal Carlens vitagram index to what were described as 'subjective responses'.

The number of papers addressing Outcomes was similar in 1985 with 14 papers reporting empirical findings. Only four papers reported cross-sectional measures of QoL in specific high salience patient populations including paraplegics in Nigeria, mental patients in nursing homes and severely mobility disabled patients. The balance of papers, however, had more emphasis on comparisons of patient groups including an increase to two clinical trials, a review (of parenteral nutrition) and a meta-analysis. Two comparisons picked up the now familiar treatment of renal disease with one of dialysis versus transplantation and one of haemodialysis versus peritoneal dialysis.

By 1990 Outcome papers had become the most common. Most of these provided simple descriptions of patient groups with various illnesses (spinal cord injury, burns, epilepsy, hypertension, cystic fibrosis, etc) including two papers which examined QoL in dying patients. In 1990 two papers also appeared that reported QoL in individual patients (rather than in diagnostic groups): one reported QoL in two patients with brain tumours and one was a more personal account of the impact of a wife's breast cancer. There were only two 'comparative' papers (comparing QoL in diabetes compared with chronic obstructive pulmonary disease and in insulin dependent diabetes compared with non-insulin dependent) and only one trial (in AIDS).

In 1995 a clear half of all papers also fell into the Outcomes category but the subtypes showed significant changes with purely descriptive papers less common, QoL in HIV and in leukaemia being the only two. Studies that compared QoL across populations were the main replacement for papers that reported the QoL of specific patient groups, though sometimes the difference was not very clear as in, for example, comparison of the QoL of patients in Texas and New York. More typically comparative studies involved measurement of QoL before and after a medical intervention (two thirds of papers in the Outcome category and 30\% of all papers in the 1995 sample). There were a further six papers with an economic focus that built on QoL measurement in developing cost-effectiveness estimates 
particularly through the calculation of Quality Adjusted Life Years (QALYs) (Williams, 1985) as a mechanism for allocation of health resources.

By 2000 almost two thirds of papers used QoL as an outcome measure. There was a trickle of descriptive papers mostly of unusual clinical populations or of non-clinical populations, such as carers' responses to hospital discharge. Most papers, however, were comparative studies, usually evaluations of interventions. Of the latter, four were trials, three more than in 1995. There were also three studies tracking changes in QoL at long-term follow-up of different diseases.

The decline in descriptive studies was maintainedin2005 (only6 out of 50). These continued exploring QoL in rare diseases (eg Systemic Lupus Erythematosis) but increasingly emphasised QoL of 'others' not directly affected by health problems such as partners of patients with cancer, carers of patients with heart failure and siblings of children with cancer. The largest group of papers in 2005 involved the use of QoL as a clinical endpoint (20 out of 50).

By 2005 QoL assessments also began to be used in experimental medicine, that is, studies in which new interventions were 'tested' on individual or small groups of patients. There had been earlier reports of QoL in individuals but these tended to be more anecdotal than methodologically rigorous but experimental medicine was a new conquest for QoL measurement. This type of study had traditionally relied on physiological surrogate outcomes to test for the signals of therapeutic benefit of a new intervention but the use of QoL as an endpoint marked a new departure. There were five such papers ranging from a report of the impact of an intervention on a rare disease in one patient to the effect of treatment of postural instability in six patients.

Since 1975 the QoL literature had been characterised by the major growth of studies measuring QoL in defined clinical and non-clinical populations (a development paralleled by the growth of useable questionnaire instruments: see below). Yet while these research studies had grown in number and proportion they had also changed in design. At the outset the task seemed to be to use the QoL measure to confirm that QoL was indeed compromised in 'at risk' populations. This process then extended to other clinical populations, the publication often being justified by the claim that no-one had previously examined the QoL of patients with XYZ disease. But this genre had a limited life: there were only so many clinical groups that could have their QoL measured so gradually the genre diminished to a trickle of papers that offered simple description of QoL in increasingly rare disease groups or in non-patient populations such as carers, spouses and siblings. Thus, just as the decline in rhetorical papers might be held to mark the end of a phase of adoption so might the brief efflorescence then virtual disappearance of simple descriptive studies mark the 'arrival' of the new concept.

In contrast the research approach that used QoL as an evaluative tool has proved more long lasting. At first this development was almost an afterthought to the earlier descriptive studies wondering whether the QoL of patients with disease $\mathrm{X}$ was better or worse than patients with disease $\mathrm{Y}$. It was but a short step then to incorporate QoL measurement into formal evaluations of an intervention using either beforeafter or comparison group designs. The pinnacle of the latter of course was the 
randomised controlled clinical trial and there was a notable increase in use of QoL as an endpoint over the period under investigation (see Figure 3).

The standardisation of QoL as an important measure of clinical outcome was also illustrated by the appearance of five empirical papers in 2005 that used QOL in innovative ways. The first examined QoL of staff-an indicator that QoL measurement was spreading not only to carers, siblings, etc, but also to health care professionals. The second paper was a study that used QoL as an independent predictor variable (for oral cancer mortality). All previous empirical papers had treated QoL as an outcome measure, as a dependent variable. The use of QoL as a predictor variable implicitly recognised the independent existence of the QoL construct. QoL was not solely a product of other forces but in itself could affect the world. The third paper reported using QoL in a clinical guideline (for the management of chronic obstructive airways disease). Guidelines had emerged over the previous decade as a means of encoding evidence-based medicine and communicating it to health professionals. The fact that QoL could be a major factor in 'selling' the guideline advice to health care professionals ('if this treatment is followed then the patient's QoL will be improved') indicated that QoL had become a widely accepted clinical outcome.

The final two papers used qualitative methods to explore QoL in two settings. The history of QoL measurement can be crudely characterised as rhetorical work around the promotion of the nascent concept followed by its stabilisation with successive measurement operations (using increasingly standardised instruments) and the consequent accumulation of a vast body of QoL 'facts'. The exploration of QoL in qualitative studies suggests recognition that the construct itself had been stabilised. It no longer needed 'fixing' with an instrument that was known to elicit its presence and 'quantity', rather the search for QoL in unstructured 'talk' implied that the construct could exist and be identified independently of any formal instrument.

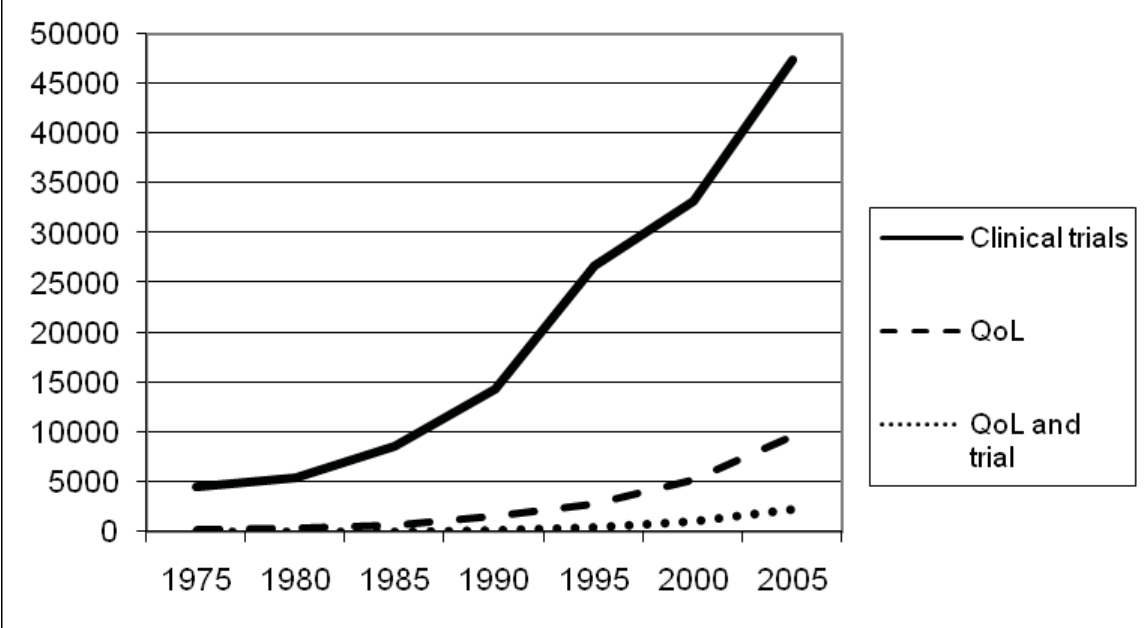

Figure 3. Citations to trials and QoL 


\section{The accumulation of Reviews}

While some of the early Non-empirical papers attempted to summarise research on QoL in a particular area it was only in 1990 that 'proto-review' papers began to appear. In that year two papers had attempted to offer an 'opinion' based on a review of the existing literature. While these 'reviews' appeared informal they did complement a further six papers (out of 50) that claimed to offer a formal review of the application of QoL in a particular field. These covered the measurement of QoL in breast cancer, in rheumatoid arthritis, in diabetes, after taking ACE inhibitors, erythropoietin for end stage renal disease and about the meaning of QoL. Five years later, in 1995, there were further five review papers; two in 2000 and three editorial reviews and seven systematic reviews in 2005.

In part the growth of these review papers reflected the emerging evidencebased medicine paradigm that gave high priority to rigorous reviews of existing evidence (particularly in the form of systematic reviews (Davidoff et al., 1995)) but in part it also indicated that the raw material of papers using QoL as an outcome were proliferating. The very appearance of these review papers indicated the successful accumulation of an empirical base for QoL measurement.

\section{The rise and fall of Methodology}

In 1980 there were four papers with a Methodology flavour. One of these described the development of a new subjective health measure (the Nottingham Health Profile), one the difficulty of measuring subjective indicators and two examined correlates of QoL in an attempt to refine what it really meant.
In 1985 the proportion of papers devoted to methodological issues doubled (8 out of 50) not counting those Miscellaneous papers that had a conceptual focus (one, for example, addressed the meaning of enjoyment). The methodological papers ranged from those developing or proposing new measures to those concerned with more general issues of bias in questionnaire based instruments.

By 1990 a quarter of the papers focused on methodological issues. Four of these tried to grapple with the conceptual nature of QoL, while the rest dealt with scale development and methods of analysis. There were similar proportions in 1995 and 2000. These latter papers were dominated by development of new questionnaires including their psychometric properties but also, in 2000, two studies reported 'head-to-head' comparisons of different instruments. Discussion of conceptual issues also continued with one paper exploring the meaning of happiness.

In 2005 there were only five explicitly methodological papers, a marked reduction from the numbers over the previous decade. Three of these reported the development of QoL measures such as testing the validity of the Kansas City Cardiomyopathy Questionnaire, translating an instrument from English into Turkish and development of a computer based QoL for the deaf. But two studies were concerned not with developing QoL measurement but with using QoL scales as means to test or validate other instruments.

The rise of Methods-papers maps the growth and consolidation of QoL measurement in evaluation research. Their proportion increased until 1985, held steady until 1995, then began to decline. Once the key measurement 
instruments were established the core task shifted from developing them to using them. Moreover the more recent appearance of methodological studies that have tried to validate non-QoL measures through their association with QoL instruments suggests the increasing stability of the latter.

\section{The growth of individual instruments}

There are currently over a 1,000 different QoL instruments in existence. Most of these are disease-specific questionnaires that can be used to map changes in QoL following treatment but many are generic instruments that can be used to assess QoL across populations irrespective of their disease profiles. The latter have become particularly important as valuefor-money considerations require health care funders to compare the impact of different treatments in different diseases.

Figure 4 shows the growth in citations of five generic instruments. The SF-36, a US originated questionnaire, clearly has come to dominate the field of generic measures. When compared to growth in disease-specific instruments, however, it shows some relative decline when set against citations to all QoL instruments (Figure 5). One of the oldest instruments, Cantril's ladder barely survives into the new century. The Sickness Impact Profile (SIP) also to some extent pre-dates the QoL era as it was originally designated as a 'subjective health measure' but despite its accumulated success it has been overtaken by the SF-36 in recent years. Of note is the 'success' of the Barthel Index which was first designed and intended as a measure of activities of daily living (ADLs) for patients with physical disabilities. As ADLs form an important component of QoL (Armstrong et al., 2007), and with the growth of physical disability of an ageing population in Western countries, its continuing use probably reflects its original purpose.

Figure 6 shows the relative recent strength (with the SIP as comparator) of two new types of instrument, economic

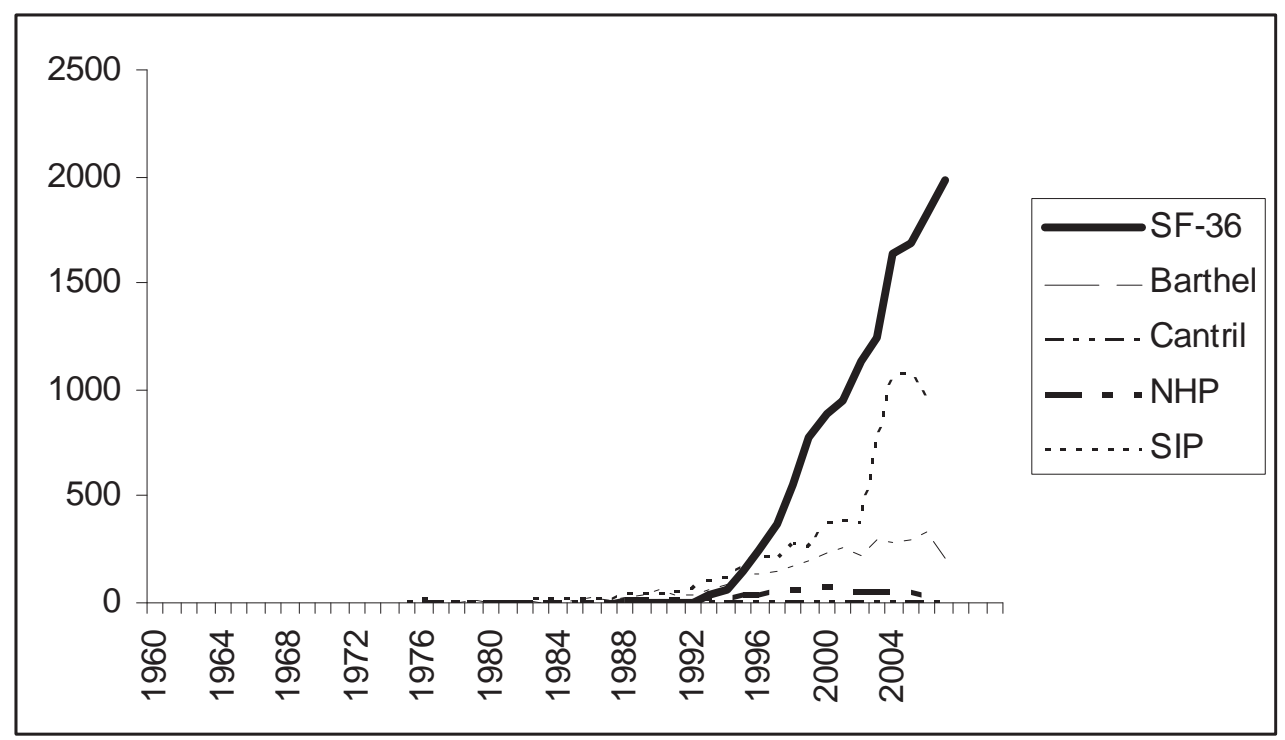

Figure 4. Citation counts showing growth of individual instruments 
and international. The two 'economic' instruments (the EQ-5D and the Health Utilities Index) have been designed for easy linkage to a cost tariff so that an economic 'value' of any QoL state can be quickly derived. Given increasing focus on efficiency on health care markets these tools have had great value and potential impact. Also noteworthy is the growth of 'international' instruments

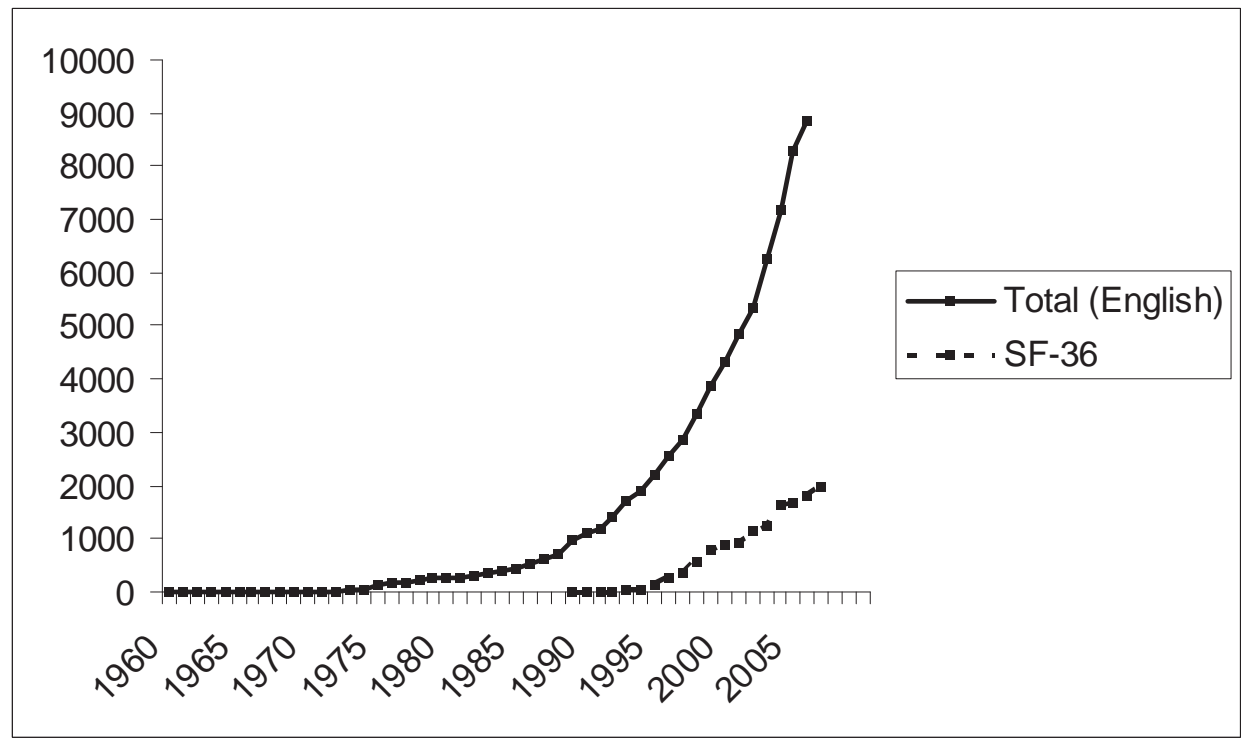

Figure 5. Citations to QoL instruments

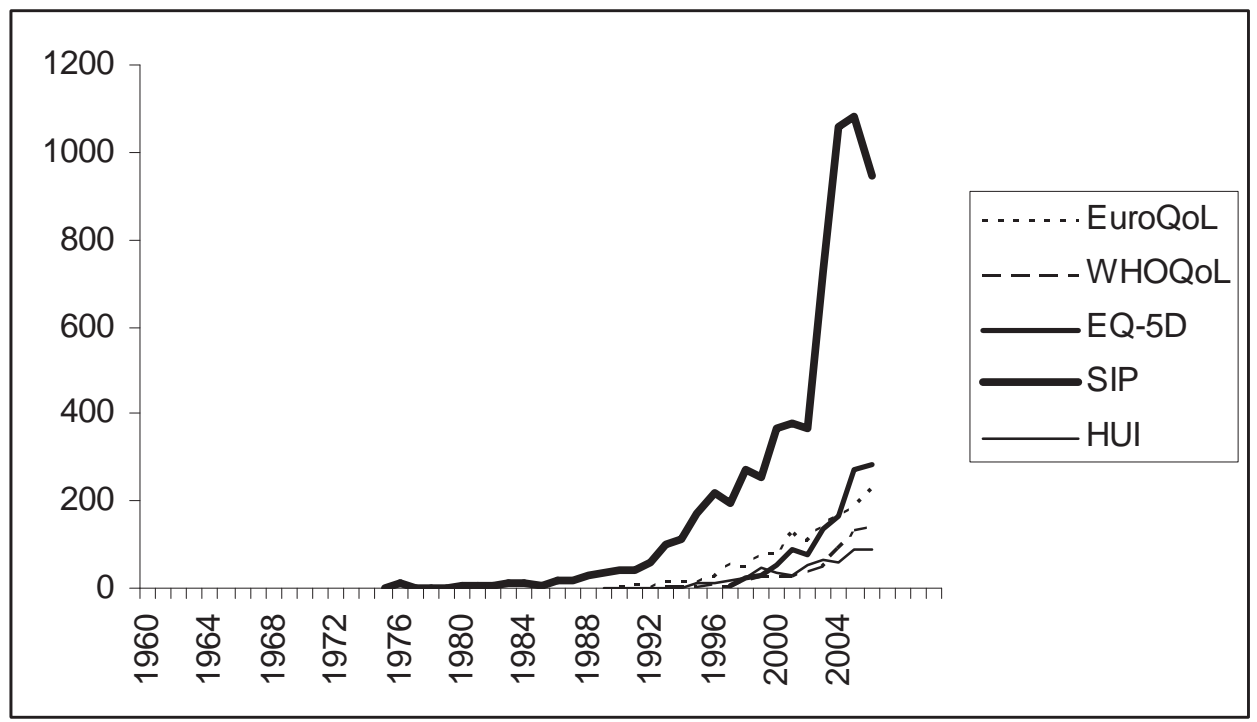

Figure 6. Growth of 'economic' and 'international' instruments 
(EuroQoL and WHOQoL) that reflects the overt need for the measurement and comparison of QoL across national boundaries by questionnaires that are not culturally specific.

\section{Discussion}

The use of a sampling technique across five year time periods seemed to provide a reasonably consistent pattern of trends across a very large number of publications. These trends, of course, have to be set against the overall growth of QoL papers (see Figure 1) such that the sample of 50 papers from 2005, say, is drawn from a much larger population of papers. Nevertheless, some broad themes can be identified despite the imprecision of some of the estimates of proportions of types of paper.

There was both a rhetorical and material genealogy of QoL standards that operated iteratively. QoL measurement started off with an advocacy period that lasted nearly 20 years. This involved promoting the concept of QoL to a naïve medical audience; towards the end of the period, however, it was often difficult to distinguish between QoL promotion and conceptual dissection as one form mutated into the other. The brief flowering of ethical studies in the late 1980s may mirror the concurrent growth in medical ethics but its absence in the later periods may reflect more on the dominance of empirical studies.

As advocacy declined a period of methodological development emerged, no doubt reflecting the general acceptance that QoL was worth measuring and could be measured with appropriate questionnaires-though discussion about what it really was continued at least until 2000. Perhaps the appearance of 'head-to-head' papers in
2000 also marks another watershed: the task of methodological research is not only to devise and test new instruments but also to destroy redundant ones so only the best survive.

Outcome studies followed closely on from the methodological development phase. These were dominated at first by simple descriptions of the QoL held by many different populations-as if researchers were both mapping the QoL landscape of medicine and playing with their powerful new device. But its increasing role was most pronounced in comparative work, in particular in evaluative studies that tried to discover whether new medical therapies and technologies really 'worked'. It is this role that dominates the use of QoL instruments in the new century.

By analysing published papers to discover the stages through which an instrument passes through - from initial conceptualisation to widespread usthe institutional and political context in which QoL developed and was applied is ignored. Even so, the discourse captured in these papers both supports and reflects those wider social contexts. On the one hand, the drive towards the evaluation of health care and assessments of comparative effectiveness which has been such an important feature of health care delivery over the last few decades, needed instruments such as QoL as their endpoints; in that sense QoL stabilisation was one of the key resources for evaluation policies. On the other hand, the increased institutional demand for viable endpoints in pursuit of efficient health care (such as the wide deployment of QALYS-quality adjusted life years - as measures of health care outcome) has underpinned the maturation of QoL measurement and the further proliferation of papers using the construct. While there has been no direct 
exploration of the wider institutional context in which QoL was embedded, the discoursedescribed in this paperhasacted as both a resource and a manifestation of activities in a wider social world beyond the documentary trail analysed here. The stabilisation of QoL through its successful operationalisation and extensive application created both the potential for new worlds of clinical practice and health policies but also constraints on those worlds. Though QoL has only recently been 'invented' it cannot be 'de-invented'; once it achieved a certain level of conceptual clarification and legitimacytogether with a wealth of standardised instruments and accumulated scores-it both described and constructed a world that was impervious to challenge. It provided new goals for modern medicine, underpinning new disciplines such as palliative care and informing decisionmaking in everyday clinical practice.

The process of stabilising QoL described here echoes previous work in the sociology of science which has its origin in Fleck's (1979) seminal work (originally published in 1935) on how scientific 'facts' are constructed. Subsequent studies, such as Latour and Woolgar's (1979) analysis of how a specific peptide was constructed as a scientific fact, have described the process of stabilisation in closer detail; indeed, more generally the scientific activity through which support is recruited to make tentative ideas into hard facts has been described by Latour (1987) as 'science-inaction'. Yet to some extent the imprecision of concepts-and their resistance to stabilisation-is also an important part of the scientific process. Rorty (1989), for example, suggested that scientific concepts expressed as metaphors had much greater creative power than when their meaning eventually became fixed through a process of 'literalisation'. Equally, in her study of the development of immunology, Lowy (1992) showed how 'loose concepts' at the boundaries of a scientific explanatory framework could facilitate stabilisation in the more central disciplinary core. QoL, however, offers another example of how stabilisation works.

In the mid 1970s QoL was only an idea, a rhetorical flourish (which mainly emerged outside of medicine) that identified a potential new outcome for medical work; thirty years later that construct has materialised as a 'real' phenomenon through the repeated application of QoL instruments that establishes and re-establishes that everyone has this 'thing' that a few decades earlier was not even a gleam in the doctor's eye. Yet despite this stabilisation there are key elements of QoL which remain contentious as there is no consensus as to what constitutes a 'good quality of life' and the continuing proliferation of instruments attests to its frequent lack of standardisation. In that sense QoL is a 'loose concept'; yet it is also a 'fact' in that it can express in precise numbers the state of a patient's well-being. It is the tension between contested concept and fixed measurement that seems to characterise QoL: broad agreement that it can now join life, death and illness as one of the central components of the Western medical paradigm, yet, at the same time, no consensus as to its precise meaning. And while it continues to be fixed as a scientific fact by a plethora of measurement instruments, there are already indications that the underlying construct may continue to shift. Recent debates about the importance of wellbeing and happiness as a goal for clinical (and other) interventions may reflect a new phase of the development of the QoL 
construct, perhaps to be renamed but to be stabilised by the application of yet more instruments and measures.

\section{References}

Armstrong, D. \& D. Caldwell (2004) 'Origins of the concept of quality of life in health care: a rhetorical solution to a political problem', Social Theory and Health 2: 361-371.

Armstrong, D., R. Lilford, J. Ogden \& S. Wessely (2007) 'Health-related quality of life and the transformation of symptoms', Sociology of Health and Illness 29: 570-583.

Brown, N. \& A. Webster (2004) New Medical Technologies and Society: Reordering Life (Cambridge: Polity Press).

Callahan, D. (1973) 'Bioethics as a Discipline', Hastings Center Studies 1: 66-73.

Davidoff, F., B. Haynes, D. Sackett \& R. Smith (1995) 'Evidence based medicine', British Medical Journal 310: 1085-6.

Fleck, L. (1979) The Genesis and Development of a Scientific Fact (Chicago: University of Chicago Press).

Jonsen, A. (1998) The birth of bioethics (New York: Oxford University Press).

Latour, B. \& S. Woolgar (1979) Laboratory Life: The Social Construction of
Scientific Facts (Beverly Hills: Sage Publications).

Latour, B. (1987) Science in Action: How to Follow Scientists and Engineers through Society (Cambridge: Harvard University Press).

Le Fanu, J. (1999) The rise and fall of modern medicine (London: Little, Brown and Co.).

Martensen, R. (2001) 'The History of Bioethics: An Essay Review', Journal of the History of Medicine and Allied Sciences 56: 168-175.

Rorty, R. (1989) Contingency, Irony, and Solidarity (Cambridge: Cambridge University Press).

Rothman, D.J. (1991) Strangers at the bedside: a history of how law and bioethics transformed medical decision making (New York: Basic Books).

Williams, A.H. (1985) 'Economics of coronary artery bypass grafting, British Medical Journal', 291: 326-329.

David Armstrong

King's College London

david.armstrong@kcl.ac.uk 